\title{
EFEITOS DO TREINAMENTO RESISTIDO SOB OS PARÂMETROS GLICÊMICOS DE INDIVÍDUOS DIABÉTICOS TIPO 2
}

\author{
Andressa Kassya Jardim Guimarães ${ }^{1}$ \\ Sandra Pereira Alves ${ }^{2}$ \\ Matheus Pereira Bastos Vieira ${ }^{3}$ \\ Gabriel Silva de Sousa ${ }^{4}$ \\ José Wilhan Cardoso Santos ${ }^{5}$
}

RESUMO: As doenças hipocinéticas se encontram em crescimento e tem acometido, principalmente os adolescentes, em razão ao estilo atual da população, dentre elas, a Diabetes Mellitus Tipo $2\left(\mathrm{DM}_{2}\right)$, que ocorre quando há uma redução na sensibilidade à insulina pelos tecidos periféricos. Isso geralmente ocorre proveniente à falha na captação de glicose, dessa forma, os níveis glicêmicos permanecem elevados. $O$ treinamento resistido pode ser utilizado como tratamento terapêutico devido aos benefícios proporcionados pela prática diária. Sendo assim, a finalidade desta pesquisa é analisar, de maneira explicativa, o treinamento resistido e sua atuação no controle glicêmico de indivíduos diabéticos tipo 2, o que foi feito através da revisão de literatura com uso de artigos científicos em língua portuguesa e língua inglesa, publicados entre os anos de 2008 e 2021. O treinamento resistido mostra ser eficiente por vários pesquisadores podendo ser adotado como profilaxia e tratamento da DM2. Sendo os efeitos da prática regular do treinamento resistido inúmeros.

Palavras-chave: Treinamento resistido. Diabetes tipo 2. Benefícios.

ABSTRACT: Hypokinetic diseases are on the rise and have mainly affected adolescents, due to the current style of the population, including Diabetes Mellitus Type 2 $\left(\mathrm{DM}_{2}\right)$, which occurs when there is a reduction in insulin sensitivity of peripheral tissues, from failure to uptake glucose, thus, blood glucose levels remain elevated. Resistance training can be used as a therapeutic treatment, due to the benefits provided by daily practice. Therefore, the purpose of this research is to analyze in an explanatory way, resistance training and its role in the glycemic control of type 2 diabetic individuals, through a literature review, covering scientific articles in Portuguese and English, published between the years of 2008 to 2021. Resistance

\footnotetext{
IAcadêmico do 8 o período do Curso de Educação Física da Faculdade Unibrás de Rio Verde.

${ }^{2}$ Acadêmico do ${ }^{8}$ o período do Curso de Educação Física da Faculdade Unibrás de Rio Verde.

${ }^{3}$ Acadêmico do $8^{\circ}$ período do Curso de Educação Física da Faculdade Unibrás de Rio Verde.

${ }^{4}$ Professor Coorientador e Membro do grupo de Estudo do Instituto Marcelo Guerra (IMG)

${ }^{5}$ Professor Orientador do Curso de Educação Fisica da Faculdade UniBrás Campus - Rio Verde wilhanxardosor3081990@gmail.com.
} 
training has been shown to be efficient by several researchers and can be adopted as a prophylaxis and treatment of DM2. Being the effects of regular practice of resistance training numerous.

Keywords: Resistance training. Type 2 diabetes. Benefits.

\section{INTRODUÇÃO}

A Diabetes Mellitus (DM) é um conjunto de desordens metabólicas, que influenciam diretamente o metabolismo da glicose, principalmente devido à falha da secreção de insulina na ação e captação de glicose, logo, sua principal característica é a hiperglicemia constante (BARROS; NUNES, 2019; SOCIEDADE BRASILEIRA DE DIABETES, 20015-2016).

A International Diabetes Federation (2015) aponta que a DM é uma das maiores causas de mortalidade na América do Sul e América Central, correspondendo a 247.500 mortes no ano de 2015, sendo o maior número de mortes no Brasil 130.700 de pessoas.

De acordo com a Sociedade Brasileira de Diabetes (2019-2020), aproximadamente 425 milhões de pessoas no mundo são portadoras de Diabetes Mellitus, sendo 90 a 95\% das ocorrências de Diabetes tipo 2. No Brasil, a prevalência da DM é de 9,0\%, ocupando o 6 o lugar entre os países de taxas mais elevadas na região das Américas.

A pesquisa em questão propôs analisar de maneira explicativa, o treinamento resistido e sua atuação no metabolismo glicêmico de indivíduos diabéticos tipo 2, e os efeitos proporcionado por intensidades diversas, tornando-o mais conhecido entre os profissionais de educação física para uma prática mais segura para esse grupo.

\section{METODOLOGIA}

Trata-se de um estudo do tipo revisão de literatura, no qual foi realizado um levantamento de estudos sobre treinamento resistido e seus benefícios para DM2. As palavras chave utilizadas para a busca de resultados deste estudo foram: treinamento resistido, diabetes mellitus tipo 2, treinamento resistido e diabetes tipo 2, tratamento 
da diabetes tipo 2. Como critérios de inclusão, foram selecionados artigos científicos em língua portuguesa e língua inglesa, publicados entre os anos de 2008 e 2021, nas bases de dados Google Acadêmico, Scientific Electronic Library Online (Scielo), PubMed e órgãos de importância na área como a Sociedade Brasileira de Diabetes e International Diabetes Federation. Foram excluídos os artigos publicados antes de 2009.

\section{FUNDAMENTAÇÃO TEÓRICA}

\section{3.r Fisiopatologia}

A DM é classificada como Diabetes Mellitus tipo I, tipo 2 e gestacional (DANILO, 2012) e essa pesquisa tem como o objeto de estudo a DM tipo 2. Os portadores dessa patologia apresentam resistência à insulina, redução na ação da insulina que impossibilita que seja usada nas células para uma captação suficiente de glicose pelos tecidos, sendo assim, a produção de glicose hepática é aumentada a fim de suprir a demanda, contribuindo ainda mais para a hiperglicemia (PÁDUA et al, 2009; BERTONHI; DIAS, 2018) acarretando deficiência da produção e secreção de insulina (ALMEIDA et al, 2014).

Sugere-se que a etiologia da doença pode estar associada à obesidade e à síndrome metabólica, representando cerca de 80 a 90\% das ocorrências (SBD, 20192020), além da disposição genética e ao meio em que estão inseridos (DE LARA, 2009); (MARASCHIN; JORGE DE FARIA et al, 2010).

Em acréscimo, quando comparados a indivíduos saudáveis, os obesos possuem menos receptores teciduais de insulina (DANILO, 2012), justificando um comprometimento à sinalização $\mathrm{da}$ insulina para os transportadores GLUT-4 resultando em uma inflamação (BARROS E NUNES, 2019).

Por ser uma doença quase (ou) silenciosa provoca danos em longo prazo em diversos órgãos, aumentando a taxa de mortalidade, sendo a principal causadora de neuropatia, doenças cardiovasculares, nefropatia, retinopatia e amputação de membros (BERTONHI; DIAS, 20I8); (SANTOS et al, 2020). Ainda há uma minoria que 
apresenta alguns sintomas, como perda de peso repentina, polidipsia, polifagia e poliúria (SBD, 2019-2020).

A DM2 pode ser diagnosticada através de exames laboratoriais de rotina, jejum $(\geq 126 \mathrm{mg} / \mathrm{dl}$ ou $7,0 \mathrm{mmol} / \mathrm{L}$ ) ou 120 minutos pós-ingestão de $75 \mathrm{~g}$ de glicose $(\geq 200$ $\mathrm{mg} / \mathrm{Dl}$ ou ir,I mmol/L) (SANTOS et al 2020). Após o diagnóstico, o tratamento deve ser feito através do uso correto de medicamentos, podendo ser antidiabéticos orais ou a insulinoterapia, ambos associados a mudanças de hábitos, incluindo dieta específica e a prática regular de atividade física. (BERTONHI; DIAS, 2018).

\section{RESULTADOS E DISCUSSÕES}

Para que os níveis de glicemia diminuam é preciso que a glicose entre nas células, sendo necessário os transportadores GLUT-4, que são ativados através da insulina. No exercício, durante a contração muscular, a AMPK é fosforizada e faz a translocação do GLUT-4 para a membrana, via essa independente de insulina (BARROS; NUNES, 2019).

O treinamento resistido tem sido estudado e apontado por autores como prevenção e intervenção não-farmacológica, pois é possível otimizar a composição corporal, metabolismo basal, densidade óssea, reduzir gordura corporal, aumentar a massa muscular e amplificar o consumo de glicose. (ZABAGLIA et al.)

A contração muscular deixa as fibras musculares mais permeáveis, facilitando a entrada da glicose circulante no músculo em ação, reduzindo os níveis de glicemia (SANTOS et al 2020). Em sua pesquisa, Liu et al (2019) aponta o aumento da massa muscular ser responsável por promover a sensibilidade a insulina. 
Tabela r. Estudos que demonstram o efeito do treinamento resistido em diabéticos tipo 2.

\begin{tabular}{|c|c|c|c|c|}
\hline Autores & População & Objetivo & Protocolo do exercício & Resultados \\
\hline $\begin{array}{l}\text { SOUZA, } \\
\text { SANTOS, } \\
\text { PARDONO } \\
(2014\end{array}$ & $\begin{array}{l}\text { homens } \\
\text { diabéticos } \\
\text { controle = Io } \\
\text { exercitado = Io }\end{array}$ & $\begin{array}{l}\text { Avaliar a redução glicêmica } \\
\text { ocasionada pelo exercício } \\
\text { resistido de alta intensidade. }\end{array}$ & $\begin{array}{l}\text { Uma única sessão de treinamento resistido, a } 75 \% \text { da carga máxima, com duração de } 24 \\
\text { minutos e } 30 \text { segundos. Todos os exercícios foram executados com } 3 \text { sets com ro repetições } \\
\text { cada. O tempo de repouso entre os sets foi de I minuto. } \\
\text { Os exercícios utilizados foram: supino reto, tríceps no pulley com barra reta, remada baixa, } \\
\text { rosca direta com barra, elevação lateral com halteres e agachamento com barra. }\end{array}$ & Redução glicêmica significante $s$ \\
\hline $\begin{array}{l}\text { DANILO, } \\
\text { MATTOS, } \\
\text { HIGINO } \\
(2012)\end{array}$ & $\begin{array}{l}8 \text { mulheres, } \\
\text { sedentárias e } \\
\text { portadoras de } \\
\text { diabetes tipo } 2\end{array}$ & $\begin{array}{l}\text { Verificar a influência do } \\
\text { treinamento resistido sobre } \\
\text { parâmetros fisiológicos, } \\
\text { antropométricos e níveis } \\
\text { séricos. }\end{array}$ & $\begin{array}{l}\text { Treinamento resistido realizado em forma de circuito, } 3 \text { vezes por semana, durante } 8 \text { semanas, } \\
\text { 3o segundos com uma carga pré-estabelecida individualmente em um teste de repetições } \\
\text { máximas e } 30 \text { segundos de intervalo. Os exercícios foram realizados em aparelhos e pesos } \\
\text { livres (anilhas e barras). Os exercícios foram realizados em uma sequência alternada } \\
\text { (membros inferiores e superiores). Foram realizados três circuitos separados por } 2 \text { minutos de } \\
\text { intervalo entre um e outro. }\end{array}$ & $\begin{array}{l}\text { Melhoras significantes do peso } \\
\text { corporal e percentual de gordura. }\end{array}$ \\
\hline $\begin{array}{l}\text { GONELA, } \\
\text { CASTRO, } \\
\text { ZANETTI } \\
(2020)\end{array}$ & 23 pacientes & $\begin{array}{l}\text { Comparar a capacidade } \\
\text { funcional e } \text { a pressão } \\
\text { arterial antes e após o } \\
\text { treinamento com pesos. }\end{array}$ & $\begin{array}{l}\text { O tempo de intervenção foi de I6 semanas, sendo I2 de treinamento e } 4 \text { de avaliações. Houve } \\
\text { aumento do volume, com o número de exercícios, e da intensidade, com o incremento de } \\
\text { pesos. } \\
\text { Nas primeiras } 13 \text { sessões, os participantes realizaram } 7 \text { exercícios (um para cada grupo } \\
\text { muscular) com } 3 \text { series ess repetições para a parte superior e } 20 \text { para parte inferior, com uma } \\
\text { intensidade de aproximadamente } 60 \% \text { de } \mathrm{IRM} \text {. } \\
\text { A intensidade foi aumentada nas I2 sessões subsequentes, com aproximadamente } 70 \% \text { de } \\
\text { IRM, } 3 \text { séries para cada exercício, com I2 repetições para membros superiores e I5 repetições } \\
\text { para membros inferiores, exceto para panturrilha e músculos adutores e abdutores, totalizando } \\
\text { 20 repetições. }\end{array}$ & $\begin{array}{l}\text { Redução significativa da pressão } \\
\text { arterial sistólica tanto em repouso } \\
\text { quanto após o esforço, força } \\
\text { muscular e a capacidade funcional. }\end{array}$ \\
\hline $\begin{array}{l}\text { BWEIR et al } \\
\text { (2009) }\end{array}$ & $\begin{array}{l}20 \text { indivíduos } \\
\text { inativos (idade } \\
\text { média de } 53,5 \\
\text { anos) com } \\
\text { diabetes tipo } 2 \\
\text { Resistido }=\text { Io } \\
\text { Esteira }=\text { Io }\end{array}$ & $\begin{array}{l}\text { Comparar os efeitos de io } \\
\text { semanas de exercícios } \\
\text { resistidos ou em esteira } \\
\text { sobre os níveis de índices } \\
\text { glicêmicos antes e } \\
\text { imediatamente após o } \\
\text { exercício. }\end{array}$ & $\begin{array}{l}\text { Os indivíduos foram pareados por idade, circunferência da cintura e sexo e atribuídos a } \\
\text { grupos de resistência isocalórica, sendo } 7 \text { exercícios para o treinamento de resistência que } \\
\text { englobava flexão / extensão de joelho e quadril, flexão / extensão de ombro, adução / } \\
\text { abdução, flexão / extensão de cotovelo e supino torácico e } 3 \text { séries de } 8 \text {-1o repetições foram } \\
\text { realizadas para todos os exercícios. E o grupo de exercícios em esteira, os participantes } \\
\text { progrediram de } 20 \text { minutos por sessão a } 60 \% \text { da frequência cardíaca máxima para } 30 \text { minutos } \\
\text { por sessão a } 75 \% \text { de sua frequência cardíaca máxima com base em protocolos estabelecidos, } \\
\text { que se reuniram } 3 \text { vezes por semana durante io semanas }\end{array}$ & \begin{tabular}{lll} 
Controle & \multicolumn{2}{c}{ glicêmico } \\
significativamente melhor em \\
adultos com diabetes tipo 2 & $\mathrm{em}$ \\
comparação com exercícios & $\mathrm{em}$ \\
esteira. &
\end{tabular} \\
\hline $\begin{array}{l}\text { MISRA et al } \\
(2008)\end{array}$ & $\begin{array}{l}30 \text { pacientes } \\
\text { indianos } \\
\text { asiáticos com } \\
\text { diabetes tipo 2, }\end{array}$ & $\begin{array}{l}\text { Avaliar o efeito do } \\
\text { protocolo de treinamento de } \\
\text { resistência progressiva na } \\
\text { sensibilidade à insulina, } \\
\text { glicemia (glicemia e níveis } \\
\text { de ArC), lipídios e } \\
\text { composição corporal. }\end{array}$ & I2 semanas de resistência progressiva de 6 grupos musculares com 2 séries eıo repetições cada. & $\begin{array}{l}\text { Resultou em melhora significativa } \\
\text { na sensibilidade à insulina, } \\
\text { glicemia, lipídios. }\end{array}$ \\
\hline
\end{tabular}

Fonte: elaboração dos autores (2021) 


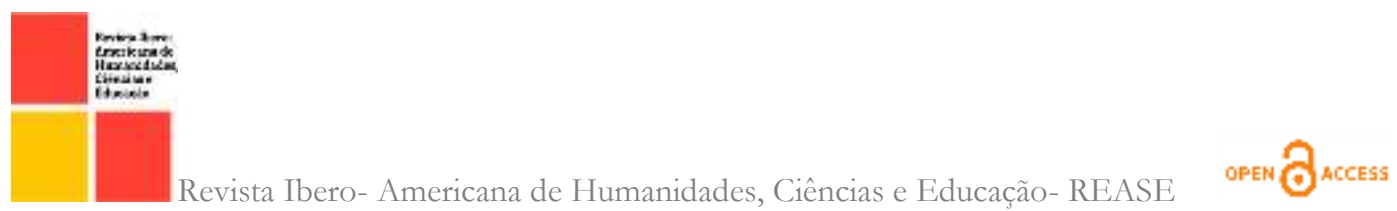

Colberg et al (2010) declara que o exercício resistido utiliza-se da glicose circulante e ácidos graxos livres como fonte de energia, produzindo um efeito hipoglicemiante e redutor do colesterol LDL, beneficiando esse grupo com a redução da mortalidade causada pelas complicações associadas ao Diabetes Mellitus 2.

Em um estudo realizado por Hsieh et al (2018), com 30 participantes diabéticos tipo 2 com idades entre 65 e 80 anos, que teve como objetivo avaliar os riscos cardiometabólicos, função muscular e desempenho físico após 12 semanas de treinamento de resistência, composto de 8 exercícios e 3 séries de 8 a 12 repetições com a intensidade $75 \%$ de IRM, foram observadas melhorias na função dos músculos e redução dos riscos de doenças cardíacas e AVC's decorrentes do DM2.

Em outra pesquisa Sousa, Santos e Pardono (2014) constataram que foi possível haver redução significativa na glicemia somente com uma única sessão de exercício resistido a 75\% de IRM, com duração de 24 minutos e 30 segundos. A amostra foi composta de 20 pessoas diabéticas do tipo 2 com mais de 40 anos do sexo masculino. Ele aponta que o aumento da atividade dos receptores e/ou de seus substratos são responsáveis por aumentar a sensibilidade à insulina.

Já em intensidade mais baixas, Takenami et al (2019) obteve em sua pesquisa de redução glicêmica, aumento de força e volume muscular, sugerindo que esses benefícios ocorreram devido a melhora na função contrátil muscular. O protocolo utilizado foi o treinamento de resistência de baixa intensidade com movimento lento e geração de força tônica, com duração de 16 semanas, sendo 2 a 3 séries de repetições máximas e a amostra composta por io idosos com diabetes tipo 2 de idade 68,2 - 9,7 anos.

No entanto, Kwon et al. (2010) apontou que não houve modificação da sensibilidade à insulina, apenas redução de gordura, aumento de força e volume muscular, em seu estudo com 28 mulheres de idade 56,4 anos +/- 7, I anos acima do peso e diabéticas tipo 2, foram submetidas ao treinamento resistido com a banda elástica com duração de 12 semanas, 3 vezes na semana e intensidade de 40 a 50\% de IRM. Cada sessão foi composta de 3 exercícios durante 6o minutos. Foi mensurada a força muscular, massa muscular, a gordura abdominal, a sensibilidade à insulina e $\mathrm{O}_{2}$ antes e depois do programa de exercícios. 


\section{CONSIDERAÇÕES FINAIS}

O treinamento resistido mostra ser eficiente por vários pesquisadores podendo ser adotado como profilaxia e tratamento da DM2. Sendo os efeitos da prática regular do treinamento resistido inúmeros, como: controle glicêmico, aumento da sensibilidade a insulina, aumento de força, volume muscular e redução das complicações associadas a patologia.

No entanto, a intensidade pode influenciar nos níveis de glicemia dos pacientes diabéticos tipo 2, fazendo-se necessário mais pesquisas para se alcançar uma intensidade mais eficiente e segura para os praticantes.

\section{REFERÊNCIAS}

DIABETES, Sociedade \& Silva Júnior, Wellington. (2019). Diretrizes da Sociedade Brasileira de Diabetes 2019-2020. Disponível em: http://www.saude.ba.gov.br/wpcontent/uploads/2020/o2/Diretrizes-Sociedade-Brasileira-de-Diabetes-2019-2020.pdf Acesso em: or abr. 2021.

DANILO DP de M, Mattos M da S, Higino WP. Efeitos do treinamento resistido em mulheres portadoras de diabetes mellitus tipo II. Rev. Bras. Ativ. Fís. Saúde [Internet]. $26^{\circ}$ de abril de 2012 [citado $8^{\circ}$ de setembro de 2021];II(2):32-8. Disponível em: https://rbafs.org.br/RBAFS/article/view/836 . Acesso em: I2 mai. 202I

DE LARA, F. N. O efeito gudo do exercicio de força e da caminhada, na glicemia de um inidviduo sedentario, diabetico do tipo 2. RBPFEX - Revista Brasileira de Prescrição e Fisiologia do Exercício, v. 3, n. 15,28 dez. 2009. Disponível em: http://www.rbpfex.com.br/index.php/rbpfex/article/view/r68 . Acesso em: or abr. 202I

ALMEIDA, S. R et al. Efeito do treinamento de força em portadores de diabetes mellitus tipo II. RBPFEX - Revista Brasileira de Prescrição e Fisiologia do Exercício, v. 8, n. 47, ıo out. 2014. Disponível em: http://www.rbpfex.com.br/index.php/rbpfex/article/view/72I . Acesso em: oI abr. 2021 
COLBERG SR, Sigal RJ, Fernhall B, et al. Exercise and type 2 diabetes: the American Association: joint position statement. Diabetes Care. 2010;33(I2):er47-er67. doi:10.2337/dcro9990. Disponível em: https://pubmed.ncbi.nlm.nih.gov/2III5758/ . Acesso em: 22 mar. 202I

BARROS, L. S. de A.; NUNES, C. da C. A influência do exercício físico na captação de glicose independente de insulina. HU Revista, [S. l.], v. 45, n. I, p. 59-64, 2019. DOI: 10.34019/1982-8047.2019.v45.2899. Disponível em: https://periodicos.ufjf.br/index.php/hurevista/article/view/2899. Acesso em: or set. 202I.

SOCIEDADE BRASILEIRA DE DIABETES. Diretrizes da Sociedade Brasileira de Diabetes 2015-2016. São Paulo, Sociedade Brasileira de Diabetes, 2016.

INTERNATIONAL DIABETES FEDERATION. IDF Diabetes atlas seventh edition. Belgium: IDF 2015, p. 50-89.

BERTONHI, Laura Gonçalves; DIAS, Juliana Chioda Ribeiro. Diabetes mellitus tipo 2: aspectos clínicos, tratamento e conduta dietoterápica. Revista Ciências Nutricionais Online, v. 2, n. 2, p. I-Io, 2018.

MARASCHIN, Jorge de Faria et al. Classificação do diabete melito. Arquivos Brasileiros de Cardiologia [online]. 2010, v. 95, n. 2 Acesso Ago 2021, pp. 40-46. Disponível em: https://doi.org/10.1590/Soo66-782X2010001200025 . Epub io Set 2010. ISSN 1678-4170. https://doi.org/10.1590/Soo66-782X2010001200025.

COlETIVO DE AUTORES. Diretrizes da Sociedade Brasileira de Diabetes: 2019 -2020. Clannad Editora, 2019-2020. Acesso io Abril 2021. Disponível em: $<$ https://www.diabetes.org.br/profissionais/images/DIRETRIZES-COMPLETA-2oI92020.pdf>. 
SANTOS, Letícia \& Souza, Lucas \& Martelli, Anderson \& Bertelli-Costa, Taiguara \& Delbim, Lucas. (2020). Treinamento resistido para pacientes diagnosticados com diabetes tipo II. Brazilian Journal of Development. 6. 7228-7239. 10.34117/bjdv6n2-139. Disponível em: https://www.brazilianjournals.com/index.php/BRJD/article/view/6873. Acesso em: ol abr. 202I.

LIU Y, Ye W, Chen Q, Zhang Y, Kuo CH, Korivi M. Resistence exercise intensity is correlated with attenuation of HbAic and insulin in patients with type 2 diabetes: a sytematic review and meta-analysis. Int J Environ Res Public Health. 2019 Jan 7;16(I):I40. doi: 10.3390/ijerphi6oroi40. PMID: 30621076; PMCID: PMC6339182. Disponível em: https://www.ncbi.nlm.nih.gov/pmc/articles/PMC6339182/. Acesso em: I2 mai. 2021.

DE SOUSA, R. A. L.; SANTOS, N. V. S.; PARDONO, E. Reducao da glicemia atraves de exercicio resistido de alta intensidade em individuos com diabtes mellitus tipo 2. RBPFEX - Revista Brasileira de Prescrição e Fisiologia do Exercício, v. 8, n. 50, 3I dez. 2014. Disponível em: < http://www.rbpfex.com.br/index.php/rbpfex/article/view/726> . Acesso em: I5 ago. 2021.

KWON HR, Han KA, Ku YH, Ahn HJ, Koo BK, Kim HC, Min KW. The effects of resistence training on muscle and body fat mass and muscle strength in type 2 diabetics women. Korean Diabetes J. 2010 Apr;34(2):I0I-Io. doi: I0.4093/kdj.2010.34.2.10I. Epub 2010 Apr 30. PMID: 20548842; PMCID: PMC2883348. Disponível em: < https://pubmed.ncbi.nlm.nih.gov/20548842/> . Acesso em: 13 abr. 2021.

ZABAGLIA, Ramon et al. Efeito dos exercicios resistidos em portadores de diabetes mellitus. CEP, $\quad$ v. 13625, p. ooo. Disponível em: http://www.rbpfex.com.br/index.php/rbpfex/article/view/207. Acesso em: 07 abr. 202I.

TAKENAMI E, Iwamoto S, Shiraishi N, Kato A, Watanabe Y, Yamada Y, Yamada S, Ishii N. Effects of low-intensity resistence training on muscular function and glycemic control in older adults with type 2 diabetes. J Diabetes Investig. 2019 Mar;10(2):331-338. doi: 
Io.III/jdi.I2926. Epub 2018 Oct I. PMID: 30175458; PMCID: PMC6400238. Disponível em: http://www.saude.ba.gov.br/wp-content/uploads/2020/o2/Diretrizes-SociedadeBrasileira-de-Diabetes-2019-2020.pdf . Acesso em: or jul. 202I.

HSIEH, Ping-Lun PT, MS I,2; Tseng, Chin-Hsiao MD, PhD3; Tseng, Yufeng Jane $\mathrm{PhD}_{4,5}$,6; Yang, Wei-Shiung $\mathrm{MD}, \mathrm{PhD}_{3,7}, 8$ Resistance Training Improves Muscle Function and Cardiometabolic Risks But Not Quality of Life in Older People With Type 2 Diabetes Mellitus: A Randomized Controlled Trial, Journal of Geriatric Physical Therapy: April/June 2018 - Volume 4I - Issue 2 - p 65-76 doi: 10.1519/JPT.oooooooooooooro7. Disponível em: https://journals.lww.com/jgpt/Fulltext/2018/o400o/Resistance_Training_Improves_Mus cle_Function_and.2.aspx . Acesso em: 04 set. 2021.

GONELA, Jefferson Thiago, Castro, Vanilde de and Zanetti, Maria Lúcia.Resistance training improves the blood pressure and functional performance of individuals with T2DM. Revista Brasileira de Medicina do Esporte [online]. 2020, v. 26, n. I Acesso 24 jun. 2021, pp. 53-57. Disponível: https://doi.org/10.1590/1517-869220202601176504 . Epub 13 Jan 2020. ISSN 1806-9940. https://doi.org/10.1590/1517-869220202601176504.

BWEIR S, Al-Jarrah M, Almalty AM, et al. Resistance exercise training lowers HbAic more than aerobic training in adults with type 2 diabetes. Diabetol Metab Syndr. 2009;1:27. Published 2009 Dec Io. doi:Io.1186/1758-5996-I-27. Disponível em: https://pubmed.ncbi.nlm.nih.gov/20003276/ . Acesso em: I8 mai. 2021.

MISRA, Anoop et al. Effect of supervised progressive resistance-exercise training protocol on insulin sensitivity, glycemia, lipids, and body composition in Asian Indians with type 2 diabetes. Diabetes care, v. 31, n. 7, p. 1282-1287, 2008. Disponível em: https://pubmed.ncbi.nlm.nih.gov/18316394/. Acesso em: 04 set. 2021.

DE SOUSA, Ricardo \& Santos, Natanael \& Pardono, Emerson. (2014). Redução da glicemia através de exercício resistido de alta intensidade em indivíduos com Diabetes 


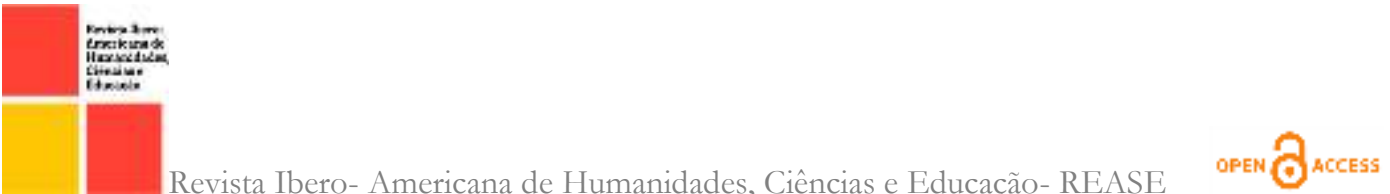

Mellitus tipo 2. Revista Brasileira de Prescrição e Fisiologia do Exercício. 8. 871-876. Disponível em: http://www.rbpfex.com.br/index.php/rbpfex/article/view/726 . Acesso em: 07 abr. 2021. 